\title{
An efficient implementation of the radial basis integral equation method
}

\author{
E. H. Ooi \& V. Popov \\ Environmental and Fluid Mechanics, Wessex Institute of Technology, \\ Southampton, $U K$
}

\begin{abstract}
In this paper, we propose an efficient implementation of the radial basis integral equation method (RBIEM) that does not involve discretization of the circular subdomains. By avoiding discretization on the boundaries of the subdomains, a major source of error in the numerical scheme can be eliminated. The proposed implementation is tested on the Helmholtz equation with higher gradients in the exact solution. Three different radial basis functions are investigated, namely the augmented thin plate spline, $r^{3}$ and $r^{4} \log (r)$. The latter two functions are augmented with the second order global polynomial. Numerical results show that the new implementation of the RBIEM produces more accurate results and is more robust in handling problems with highly variable solutions. By avoiding the boundary discretization, the tasks of keeping track of the boundary elements and the boundary nodes are not needed, which can be a daunting task especially in three-dimensional problems with complicated geometries. The proposed implementation of the RBIEM is promising and the feasibility of the approach in three-dimensional problems is currently being investigated.

Keywords: meshless, radial basis function, discretization, Helmholtz, efficiency.
\end{abstract}

\section{Introduction}

In the past decade, research in numerical methods has been moving towards meshless approaches for solving partial differential equations. In particular, meshless methods based on integral equations have been gaining wide attention due to the accuracy of the integral equation based methods and the reduced requirements for meshing. Meshless methods based on integral equations such as the local boundary integral equation (LBIE) method developed by Zhu et 
al. [1, 2] have been successfully used to solve elasticity [3] and computational fluid dynamics [4] problems. While the LBIE may be considered to be a meshless approach, its implementation requires integration over part of the global boundary when the collocation nodes are located on the boundary of the solution domain. From a conceptual point of view, the approach is not truly meshless.

Popov and Bui [5] introduced the radial basis integral equation method (RBIEM), which is a meshless method based on the idea of the dual reciprocity method-multi domain (DRM-MD) with overlapping subdomains. Similar to the LBIE, the RBIEM generates around each node, a circular subdomain. The generation of the circular subdomains are by no means, the same as the domain discretization involved in the multi-domain method since there are no restrictions on how the subdomains are created. Generally, the subdomains may overlap one another and may even extend beyond the global boundary. Unlike the LBIE however, the RBIEM does not involve integration over the global boundary. All boundary integrations are performed only on the boundaries of the circular subdomains, making the RBIEM a truly meshless approach. Essentially, the RBIEM may be viewed as a meshless implementation of the DRM-MD with overlapping subdomains.

In the RBIEM [5], the boundary of each subdomain is discretized into quadratic elements, where the boundary integrals are evaluated numerically for each element. Unknown field variables on the nodes of each boundary element are interpolated using neighbouring nodes based on the radial basis functions (RBFs). Since the governing integral equations in the RBIEM are defined over each circular subdomain, it is perhaps more practical to transform the integration domain into polar coordinates and to carry out the integration numerically without involving any boundary discretization. By avoiding the discretization on the boundary of each subdomain, it is possible to improve on the accuracy of the numerical scheme.

The objective of this paper is to compare the performance of the proposed implementation of the RBIEM, whereby the boundary discretization of each subdomain is avoided, with the conventional RBIEM. The numerical schemes are tested using three different RBFs, namely $r^{2} \log (r), r^{3}$ and $r^{4} \log (r)$. The first function is augmented with a global first order polynomial, while the latter two functions are augmented with second order polynomial. The choice of using $r^{3}$ and $r^{4} \log (r)$ is motivated by the study carried out by Partridge [8].

For simplicity, the proposed efficient implementation of the RBIEM in this paper is hereafter, referred to as RBIEM-I.

\section{Mathematical formulations}

\subsection{The radial basis integral equation method}

Consider the following partial differential equation in the two-dimensional region $R$ enclosed by the surface $S$ :

$$
\nabla^{2} u(r)=f, \quad \text { for } r \in R \cup S
$$


where $u(r)$ is the potential variable, $r$ is the coordinates in the Cartesian coordinate system and $f$ is the non-homogeneous term. Equation (1) is subjected to the following boundary conditions:

$$
\begin{aligned}
u(r) & =u_{o} \quad \text { at } S_{1} \\
\frac{\partial}{\partial n} u(r) & =q_{o} \quad \text { at } S_{2}
\end{aligned}
$$

where $S_{1} \cup S_{2}=S$ and $u_{\mathrm{o}}$ and $q_{\mathrm{o}}$ are suitably prescribed functions. To obtain a solution of (1) subjected to the boundary conditions in (2) using the RBIEM, a set of $N_{t}$ nodes denoted by $\xi_{i}$, for $i=1,2, \ldots, N_{t}-1, N_{t}$, distributed across the boundary and interior of the solution domain, is selected. This is illustrated in Figure 1a.

A circular subdomain, $R_{i}$ enclosed by the boundary $S_{i}$, centred about the point $\xi_{i}$ is generated for each node. This is shown in Figure 1b. In principle, the subdomains can be of any shape, although the use of a circular one simplifies the generation of boundaries and the evaluation of the integrals.

a.

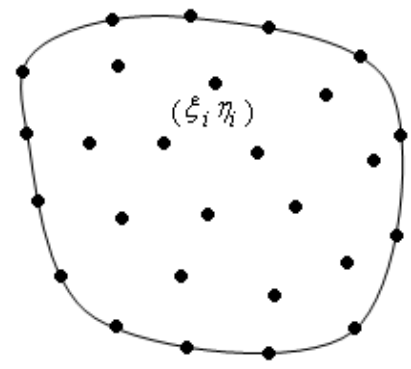

b.

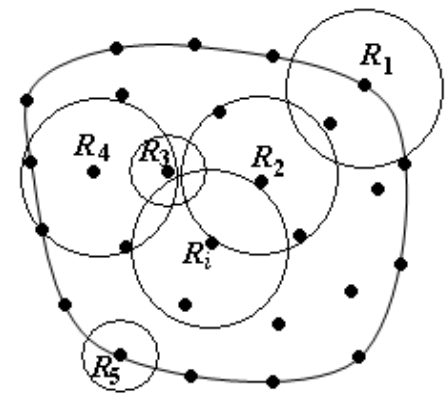

Figure 1: a) Distribution of nodes on the boundary and interior, and b) representation of some of the circular subdomains with different radii generated around the collocation nodes.

Once the collocation nodes are selected, the integral equations describing the potential and its derivatives in the $x$ - and $y$-directions, respectively over each subdomain are derived, which hold in each subdomain. Using the Green's second identity, we obtain

$$
\begin{aligned}
\left.u(r)\right|_{r=\xi}= & \int_{S_{i}} u(r) \frac{\partial}{\partial n} \Phi(r, \xi) d s(r)-\int_{S_{i}} n_{x}(r) \Phi(r, \xi) \frac{\partial}{\partial x} u(r) d s(r) \\
& -\int_{S_{i}} n_{y}(r) \Phi(r, \xi) \frac{\partial}{\partial y} u(r) d s(r)+\iint_{R_{i}} f_{i} \cdot \Phi(r, \xi) d R(r),
\end{aligned}
$$

and differentiating (3) with respect to $x$ and $y$, yields 


$$
\begin{aligned}
\left.\frac{\partial u(r)}{\partial x}\right|_{r=\xi} & =\int_{S_{i}} u(r) \frac{\partial^{2}}{\partial x \partial n} \Phi(r, \xi) d s(r)-\int_{S_{i}} n_{x}(r) \frac{\partial}{\partial x} \Phi(r, \xi) \frac{\partial}{\partial x} u(r) d s(r) \\
& -\int_{S_{i}} n_{y}(r) \frac{\partial}{\partial x} \Phi(r, \xi) \frac{\partial}{\partial y} u(r) d s(r)+\frac{\partial}{\partial x} \iint_{R_{i}} f_{i} \cdot \Phi(r, \xi) d R(r),
\end{aligned}
$$

and

$$
\begin{aligned}
\left.\frac{\partial u(r)}{\partial y}\right|_{r=\xi} & =\int_{S_{i}} u(r) \frac{\partial^{2}}{\partial y \partial n} \Phi(r, \xi) d s(r)-\int_{S_{i}} n_{x}(r) \frac{\partial}{\partial y} \Phi(r, \xi) \frac{\partial}{\partial x} u(r) d s(r) \\
& -\int_{S_{i}} n_{y}(r) \frac{\partial}{\partial y} \Phi(r, \xi) \frac{\partial}{\partial y} u(r) d s(r)+\frac{\partial}{\partial y} \iint_{R_{i}} f_{i} \cdot \Phi(r, \xi) d R(r),
\end{aligned}
$$

where $d s$ is the length of an infinitesimal part of $S, d R$ is the area of an infinitesimal region of $R$ and $\Phi(r, \xi)$ is the fundamental solution of the Laplace equation, which is given by

$$
\Phi(r, \xi)=\frac{1}{2 \pi} \ln |r-\xi|
$$

In writing (3) to (5), the following relation

$$
\frac{\partial u(r)}{\partial n}=n_{x}(r) \frac{\partial u(r)}{\partial x}+n_{y}(r) \frac{\partial u(r)}{\partial y},
$$

has been utilized, where $n_{x}$ and $n_{y}$ are the components of the outward unit normal vector in the $x$ - and $y$-directions, respectively.

The RBIEM employs discretization over the boundaries of each subdomain. For more details one may refer to Popov and Bui [5]. In our proposed formulation, no discretization of the boundaries of the subdomains is involved. Instead, we approximate the unknown variables with RBFs such that

$$
\phi(r)=\sum_{j=1}^{N} \rho\left(r, r_{j}\right) \alpha_{j},
$$

where $\phi(r)$ is the unknown variable represented by $u(r), \partial u(r) / \partial x$ and $\partial u(r) / \partial y$, $\rho\left(r, r_{j}\right)$ are the RBFs, $\alpha_{j}$ are unknown coefficients and $N$ is the number of approximation points $r_{j}$ in the neighbourhood of the integration node. The unknown coefficients may be expressed in terms of nodal values such that

$$
\alpha_{j}=\sum_{j=1}^{N} W_{m j} \phi_{j}, \quad \text { for } m=1,2, \ldots, N-1, N .
$$

where $W_{m j}$ are explicitly given by 


$$
\sum_{j=1}^{N} W_{k j} \rho\left(r_{j}, r_{m}\right)=\left\{\begin{array}{l}
0, \text { if } k \neq m \\
1, \text { if } k=m
\end{array} ; \text { for } j, m=1,2, \ldots, N-1, N .\right.
$$

Substituting (8) and (9) into (3) to (5), we obtain

$$
\begin{aligned}
\left.u(r)\right|_{r=\xi} & =\sum_{j=1}^{N} u_{j} \sum_{k=1}^{N} W_{k j} F_{j}-\sum_{j=1}^{N} \frac{\partial u_{j}}{\partial x} \sum_{k=1}^{N} W_{k j} G_{j}-\sum_{j=1}^{N} \frac{\partial u_{j}}{\partial y} \sum_{k=1}^{N} W_{k j} H_{j} \\
& +\iint_{R_{i}} f_{i} \cdot \Phi(r, \xi) d R(r), \\
\left.\frac{\partial u(r)}{\partial x}\right|_{r=\xi}= & \sum_{j=1}^{N} u_{j} \sum_{k=1}^{N} W_{k j} \frac{\partial F_{j}}{\partial x}-\sum_{j=1}^{N} \frac{\partial u_{j}}{\partial x} \sum_{k=1}^{N} W_{k j} \frac{\partial G_{j}}{\partial x}-\sum_{j=1}^{N} \frac{\partial u_{j}}{\partial y} \sum_{k=1}^{N} W_{k j} \frac{\partial H_{j}}{\partial x} \\
& +\frac{\partial}{\partial x} \iint_{R_{i}} f_{i} \cdot \Phi(r, \xi) d R(r),
\end{aligned}
$$

and

$$
\begin{aligned}
\left.\frac{\partial u(r)}{\partial y}\right|_{r=\xi} & =\sum_{j=1}^{N} u_{j} \sum_{k=1}^{N} W_{k j} \frac{\partial F_{j}}{\partial y}-\sum_{j=1}^{N} \frac{\partial u_{j}}{\partial x} \sum_{k=1}^{N} W_{k j} \frac{\partial G_{j}}{\partial y}-\sum_{j=1}^{N} \frac{\partial u_{j}}{\partial y} \sum_{k=1}^{N} W_{k j} \frac{\partial H_{j}}{\partial y} \\
& +\frac{\partial}{\partial y} \iint_{R_{i}} f_{i} \cdot \Phi(r, \xi) d R(r),
\end{aligned}
$$

where $F_{j}, G_{j}$ and $H_{j}$ are coefficients associated with the boundary integrals.

\subsection{The dual reciprocity method}

The domain integrals in (11) to (13) may be treated using, e.g., direct evaluation of the domain integral or the DRM [6]. In this case the DRM is employed, which converts the domain integral into an equivalent boundary integral using approximation of the non-homogeneous term $f$, see (1), based on the RBFs and the Green's second identity. By applying the DRM, (11) to (13) can be reformulated as:

$$
\begin{aligned}
\left.u(r)\right|_{r=\xi} & =\sum_{j=1}^{N} u_{j} \sum_{k=1}^{N} W_{k j} F_{j}-\sum_{j=1}^{N} \frac{\partial u_{j}}{\partial x} \sum_{k=1}^{N} W_{k j} G_{j}-\sum_{j=1}^{N} \frac{\partial u_{j}}{\partial y} \sum_{k=1}^{N} W_{k j} H_{j} \\
& +\sum_{j=1}^{N} f_{j} \sum_{k=1}^{N} W_{k j} \Psi_{j}, \\
\left.\frac{\partial u(r)}{\partial x}\right|_{r=\xi}= & \sum_{j=1}^{N} u_{j} \sum_{k=1}^{N} W_{k j} \frac{\partial F_{j}}{\partial x}-\sum_{j=1}^{N} \frac{\partial u_{j}}{\partial x} \sum_{k=1}^{N} W_{k j} \frac{\partial G_{j}}{\partial x}-\sum_{j=1}^{N} \frac{\partial u_{j}}{\partial y} \sum_{k=1}^{N} W_{k j} \frac{\partial H_{j}}{\partial x} \\
& +\sum_{j=1}^{N} f_{j} \sum_{k=1}^{N} W_{k j} \frac{\partial \Psi_{j}}{\partial x},
\end{aligned}
$$

and 


$$
\begin{aligned}
\left.\frac{\partial u(r)}{\partial y}\right|_{r=\xi} & =\sum_{j=1}^{N} u_{j} \sum_{k=1}^{N} W_{k j} \frac{\partial F_{j}}{\partial y}-\sum_{j=1}^{N} \frac{\partial u_{j}}{\partial x} \sum_{k=1}^{N} W_{k j} \frac{\partial G_{j}}{\partial y}-\sum_{j=1}^{N} \frac{\partial u_{j}}{\partial y} \sum_{k=1}^{N} W_{k j} \frac{\partial H_{j}}{\partial y} \\
& +\sum_{j=1}^{N} f_{j} \sum_{k=1}^{N} W_{k j} \frac{\partial \Psi_{j}}{\partial y},
\end{aligned}
$$

where $\Psi_{j}$ is defined as

$$
\Psi_{j}=\left.\hat{u}(r)\right|_{r=\xi}-\int_{S_{i}} \hat{u}\left(r, r_{j}\right) \frac{\partial}{\partial n} \Phi(r, \xi) d s(r)+\int_{S_{i}} \Phi(r, \xi) \frac{\partial}{\partial n} \hat{u}\left(r, r_{j}\right) d s(r),
$$

where $\hat{u}$ is the particular solution associated with the RBFs. For more details on the implementation of the DRM, one may refer to Partridge et al. [7].

\section{Numerical implementation}

To implement the RBIEM, (14) to (16) are collocated on the $N_{t}$ nodes distributed across the boundary and interior of the solution domain. When the collocation node is located at the interior, all three equations are used in constructing the system of linear algebraic equations. When the collocation node is located on the boundary where potential is specified, only (15) and (16) are required. When the collocation node is located on the boundary where the normal derivative of potential is specified, two cases apply. When the normal derivative of potential is zero, the derivative in the direction closer to the unit normal is eliminated using (7) and the remaining two equations are used to assemble the system of linear algebraic equations. When the normal derivative of potential is not zero, the same procedure applies except that the derivative that is closer to the boundary is eliminated [5].

In the present formulation, the same set of nodes is chosen for both the interpolation of field variables and for the DRM. In this case, the $N$ points closest to the centre of the subdomain are selected. This step simplifies the search for the neighbouring nodes, saves the overall computational time of the numerical model and ensures that all the RBF approximations are performed using the same number of nodes [5].

All boundary integrals in the proposed formulation are transformed into the polar coordinate system and are calculated numerically over the sub-domains using the Gaussian quadrature, without discretisation of the boundaries of the sub-domains.

\section{Numerical examples}

The performance of the proposed numerical scheme is compared with the conventional implementation of the RBIEM by solving the Helmholtz equation

$$
\nabla^{2} u(x, y)+\kappa^{2} u(x, y)=0,
$$


where $\kappa$ is a pre-defined positive real number, which is also known as the wavenumber. The problem is defined over a square domain bounded by the limits $-0.5 \leq x \leq 0.5$ and $-0.5 \leq y \leq 0.5$ and subjected to the following boundary conditions:

$$
\begin{gathered}
u( \pm 0.5, y)=\sin \left( \pm \frac{\kappa}{2 \sqrt{2}}\right) \sin \left(\frac{\kappa}{\sqrt{2}} y\right), \\
\frac{\partial}{\partial n} u(x, \pm 0.5)= \pm \frac{\kappa}{\sqrt{2}} \cos \left( \pm \frac{\kappa}{2 \sqrt{2}}\right) \sin \left(\frac{\kappa}{\sqrt{2}} x\right),
\end{gathered}
$$

The exact solution of this problem is given by

$$
u(x, y)=\sin \left(\frac{\kappa}{\sqrt{2}} x\right) \sin \left(\frac{\kappa}{\sqrt{2}} y\right) .
$$

Two values of $\kappa$ are considered, namely 5 and 10 , where the distributions of the exact solution across the solution domain for each $\kappa$ are illustrated in Figures $2 \mathrm{a}$ and $2 \mathrm{~b}$, respectively. In RBIEM, 16 continuous quadratic boundary elements are discretized on the boundary of each subdomain. Both numerical schemes employ 25 nodes in the RBFs approximations of the potential and derivatives over the local boundaries.
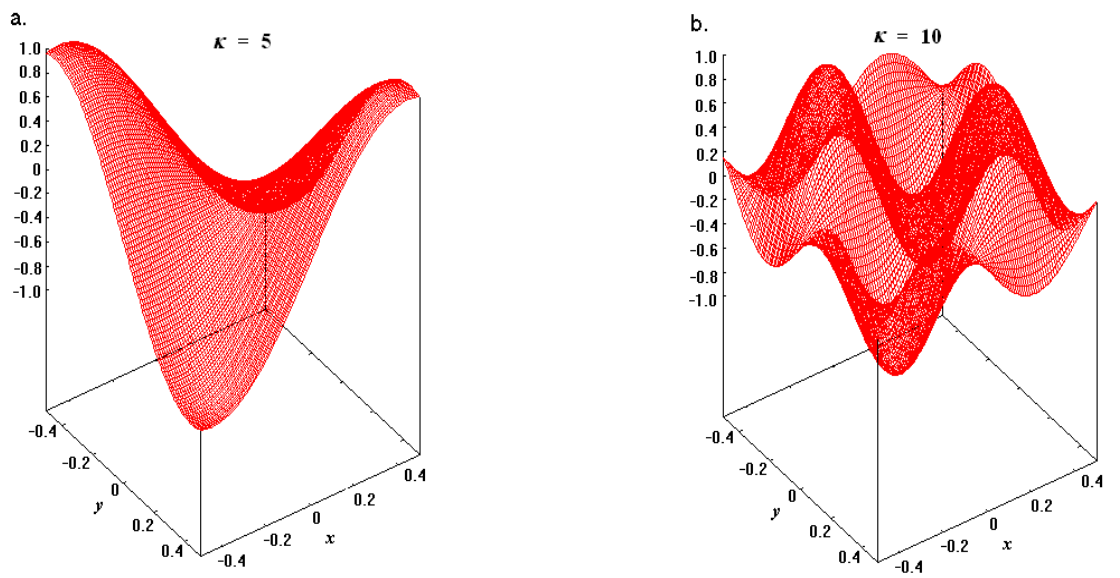

Figure 2: The exact solution of the Helmholtz problem for a) $\kappa=5$ and b) $\kappa=$ 10.

All numerical computations are coded using the programming language FORTRAN. The built-in sparse solver, PARDISO is used to solve the assembled sparse system of linear algebraic equations. A desktop computer with $2.61 \mathrm{GHz}$ AMD Athlon 64, X2 Dual Processor and 2.0GB of RAM is used for all computations. 
Results are presented in Table 1 for five different sets of nodes, namely $N_{t}=$ 2601, 3721, 6561, 8281 and 10201. Of the three RBFs tested, the worst results are obtained when the ATPS is used. The accuracies of both functions $r^{3}$ and $r^{4} \log (r)$ are comparable and they are by one order of magnitude more accurate than the results obtained using the ATPS. From Table 1, it is apparent that the RBIEM-I produces results that are more accurate than the RBIEM, as indicated by the lower $L_{2}$ norm errors. Significant difference in accuracy between RBIEM and RBIEM-I are obtained only when the functions $r^{3}$ and $r^{4} \log (r)$ are used. Unlike the RBIEM-I, no significant improvement in the accuracy of the RBIEM is observed when the number of nodes increases beyond 6561 ; indicating a higher convergence rate in the RBIEM-I case.

Table 1: $\quad L_{2}$ norm errors $(\%)$ for the Helmholtz problem when $\kappa=5$.

\begin{tabular}{lcccccc}
\hline$N_{t}$ & \multicolumn{2}{c}{ ATPS } & \multicolumn{2}{c}{$r^{3}$} & \multicolumn{2}{c}{$r^{4} \log (r)$} \\
& RBIEM & RBIEM-I & RBIEM & RBIEM-I & RBIEM & RBIEM-I \\
\hline$u$ & & & & & & \\
2601 & 0.2235 & 0.2261 & 0.0137 & 0.0092 & 0.0140 & 0.0094 \\
3721 & 0.1974 & 0.1964 & 0.0112 & 0.0054 & 0.0115 & 0.0059 \\
6561 & 0.1704 & 0.1694 & 0.0099 & 0.0024 & 0.0101 & 0.0029 \\
8281 & 0.1615 & 0.1605 & 0.0097 & 0.0017 & 0.0097 & 0.0022 \\
10201 & 0.1552 & 0.1542 & 0.0096 & 0.0012 & 0.0097 & 0.0017 \\
& & & & & & \\
$\partial u / \partial x$ & & & & & & \\
2601 & 0.4348 & 0.4330 & 0.0326 & 0.0191 & 0.0319 & 0.0172 \\
3721 & 0.3813 & 0.3790 & 0.0281 & 0.0114 & 0.0282 & 0.0108 \\
6561 & 0.3298 & 0.3271 & 0.0255 & 0.0052 & 0.0258 & 0.0053 \\
8281 & 0.3138 & 0.3109 & 0.0251 & 0.0037 & 0.0253 & 0.0040 \\
10201 & 0.3027 & 0.2998 & 0.0248 & 0.0028 & 0.0251 & 0.0032 \\
& & & & & & \\
$\partial u / \partial y$ & & & & & & \\
2601 & 0.2318 & 0.2342 & 0.0145 & 0.0138 & 0.0111 & 0.0092 \\
3721 & 0.2004 & 0.2029 & 0.0106 & 0.0085 & 0.0090 & 0.0057 \\
6561 & 0.1688 & 0.1713 & 0.0086 & 0.0041 & 0.0084 & 0.0028 \\
8281 & 0.1589 & 0.1614 & 0.0084 & 0.0030 & 0.0083 & 0.0020 \\
10201 & 0.1519 & 0.1544 & 0.0083 & 0.0023 & 0.0083 & 0.0016 \\
\hline \hline
\end{tabular}

Figure 3 compares the distribution of percentage error between the RBIEM and RBIEM-I for $\kappa=10$ obtained using the function $r^{4} \log (r)$ with 10201 nodes.

Both numerical schemes are found to produce mean percentage errors that are less than $1 \%$. However, the RBIEM produces peak percentage errors that are greater than $10 \%$. In the case of $\partial u / \partial x$, the maximum percentage error is $121 \%$. Although peak errors are also observed in the RBIEM-I, the magnitudes are one order of magnitude lower than those obtained using the RBIEM. 
a. RBIEM

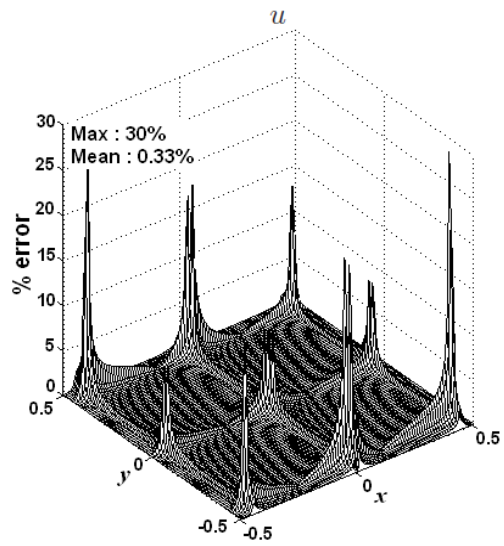

$\partial u / \partial x$

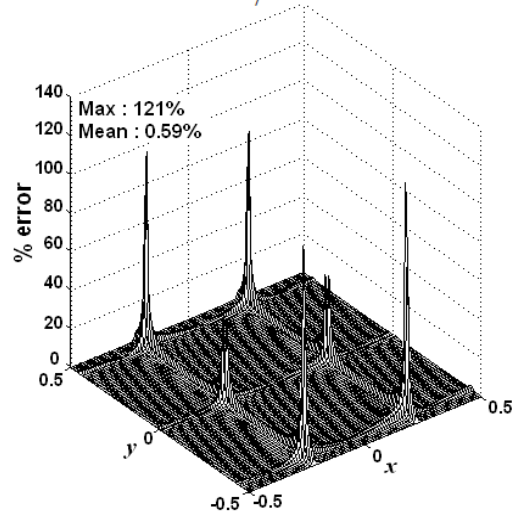

$\partial u / \partial y$

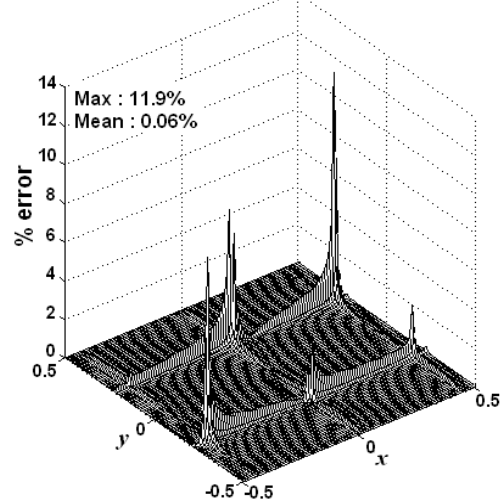

b. RBIEM-I

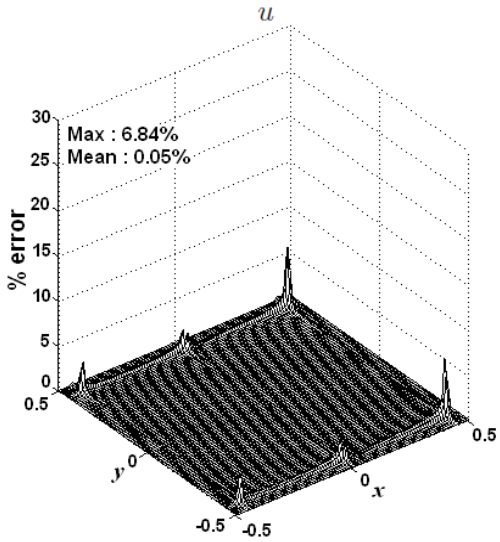

$\partial u / \partial x$

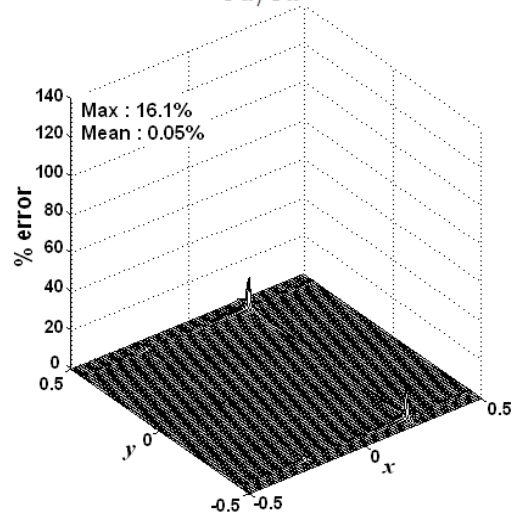

$\partial u / \partial y$

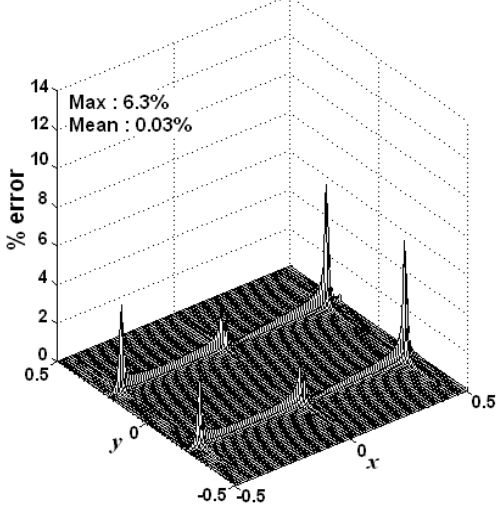

Figure 3: Distribution of percentage errors of $u, \partial u / \partial x$ and $\partial u / \partial y$ for $\kappa=10$ obtained using a) RBIEM and b) RBIEM-I. 


\section{Discussions}

From the numerical example presented, the RBIEM-I is found to be more accurate and more robust than the RBIEM. By avoiding discretization of the circular subdomain, a major source of error is eliminated. Significant improvement in the results are only observed when the functions $r^{3}$ and the $r^{4} \log (r)$ are used. When the ATPS is used, the accuracies of both the RBIEM and RBIEM-I are comparable. Since the ATPS produces the least accurate results among the three RBFs investigated, the small difference in the numerical accuracy when ATPS is used is perhaps caused by the errors from the ATPS approximation, which make any accuracy increase due to the RBIEM-I approach seem insignificant.

Both numerical schemes show convergence as the number of nodes increases. However, the increase in the number of nodes used increases the number of elements used in discretisations of the local sub-domains in the RBIEM, which further increases the numerical errors. This explains the slow convergence of the solution for RBIEM beyond 6561 nodes.

The RBIEM-I handles problems with high gradients in the solution better than the RBIEM. For problems with less variation in the solution, such as for the case of $\kappa=5$, the RBIEM is capable of producing results that are relatively accurate. As the value of $\kappa$ increases, the numerical scheme becomes unstable and fails to capture accurately the solution in areas where the gradients are large. This is indicated by the peak percentage errors, which are localized to regions where the gradients of $u, \partial u / \partial x$ and $\partial u / \partial y$ are the highest.

The same behaviour is also observed in the RBIEM-I, although this scheme is less sensitive towards the increase in $\kappa$. This indicates the robustness of the RBIEM-I.

An additional benefit of the RBIEM-I is that there is no need to keep track of the node and element numbering on the local sub-domain since no discretization is involved. While this may not be an issue in two-dimensional problems, the task of keeping track of the boundary nodes and elements in three-dimensional problems is more rigorous, especially in problems with complicated geometry. In three-dimensional problems, the RBIEM-I can be implemented by expressing the integration domain in the spherical coordinates system. Hence, one only needs to know the radius of each spherical subdomain and its centroid when performing the integration over the boundaries. The implementation of the RBIEM-I in three-dimensional problems is currently being investigated.

\section{Conclusions}

An efficient implementation of the RBIEM, which avoids the discretization of the boundaries of the subdomains, has been proposed. The proposed implementation is shown to be very accurate and stable even with only 20 Gaussian integration points. The new formulation is easier to implement and may be particularly useful in solving three-dimensional problems. 


\section{References}

[1] Zhu T, Zhang J.D. and Atluri S.N., A local boundary integral equation (LBIE) method in computational mechanics, and a meshless discretization approach, Computational Mechanics, 1988; 21: 223-235.

[2] Zhu T, Zhang J.D. and Atluri S.N., A meshless local boundary integral equation (LBIE) method for solving nonlinear problems, Computational Mechanics, 1998; 22: 174-186.

[3] Sladek J., Sladek V. and Atluri S.N., Local boundary integral equation (LBIE) method for solving problems of elasticity with nonhomogeneous material properties, Computational Mechanics, 2000; 24: 456-462.

[4] Sellountos E.J. and Sequeira A., An advanced meshless LBIE/RBF method for solving two-dimensional incompressible fluid flows, Computational Mechanics, 2008; 41: 617-631.

[5] Popov V. and Bui T.T., A meshless solution to two-dimensional convectiondiffusion problems, Engineering Analysis with Boundary Elements, 2010; 34: 680-689.

[6] Nardini D. and Brebbia C., A new approach in solid mechanics by an alternative boundary element procedure, International Journal of Soil Dynamics and Earthquake Engineering, 1983; 2: 228-233.

[7] Partridge, P.W., Brebbia, C.A. and Wrobel, L.C., The Dual Reciprocity Boundary Element Method. Computational Mechanics Publication, Southampton, 1992.

[8] Partridge P.W., Towards criteria for selecting approximation functions in the dual reciprocity method, Engineering Analysis with Boundary Elements, 2000; 24: 519-529. 